Algebraic $8 \mathcal{G}$ Geometric Topology

Volume 2 (2002) 157-170

Published: 6 March 2002

ATG

\title{
Abelian Subgroups of the Torelli Group
}

\author{
William R. VAUtaW
}

\begin{abstract}
Let $\mathbf{S}$ be a closed oriented surface of genus $g \geq 2$, and let $\mathcal{T}$ denote its Torelli group. First, given a set $\mathfrak{E}$ of homotopically nontrivial, pairwise disjoint, pairwise nonisotopic simple closed curves on $\mathbf{S}$, we determine precisely when a multitwist on $\mathfrak{E}$ is an element of $\mathcal{T}$ by defining an equivalence relation on $\mathfrak{E}$ and then applying graph theory. Second, we prove that an arbitrary Abelian subgroup of $\mathcal{T}$ has rank $\leq 2 g-3$.
\end{abstract}

AMS Classification 57M60; 20F38

Keywords Mapping class group, Torelli group, multitwist

\section{Introduction}

Here we present the notation, definitions, and terminology that will be used in the paper.

\subsection{Surfaces}

Throughout this work, $\mathbf{S}$ will denote a closed, connected, oriented surface. We use the symbols $\mathfrak{a}, \mathfrak{b}, \mathfrak{c}, \mathfrak{e}, \mathfrak{h}$ to denote simple closed curves on $\mathbf{S}$.

The mapping class group, $\mathcal{M}(\mathbf{S})$, of $\mathbf{S}$ is the group of isotopy classes of orientation preserving self-homeomorphisms of $\mathbf{S}$. In general, we will not distinguish between a map $f: \mathbf{S} \rightarrow \mathbf{S}$ and its isotopy class. The symbol $D_{\mathfrak{e}}$ will denote the right Dehn twist about the simple closed curve $\mathfrak{e}$. Recall that if $\mathfrak{a}$ and $\mathfrak{b}$ are simple closed oriented curves on $\mathbf{S}$, then in $H_{1}(\mathbf{S})$, the first homology group of $\mathbf{S}$ with integer coefficients, we have

$$
D_{\mathfrak{a}}(\mathfrak{b})=\mathfrak{b}+\langle\mathfrak{a}, \mathfrak{b}\rangle \mathfrak{a}
$$

where $\langle\mathfrak{a}, \mathfrak{b}\rangle$ denotes the algebraic intersection number of $\mathfrak{a}$ and $\mathfrak{b}$. Also, the Dehn twists $D_{\mathfrak{a}_{1}}$ and $D_{\mathfrak{a}_{2}}$ commute if and only if the isotopy classes of the curves $\mathfrak{a}_{1}$ and $\mathfrak{a}_{2}$ have representatives that are disjoint. 
The Torelli group, $\mathcal{T}=\mathcal{T}(\mathbf{S})$, of $\mathbf{S}$ is the subgroup of the mapping class group consisting of the isotopy classes of those self-homeomorphisms of $\mathbf{S}$ which induce the identity isomorphism on $H_{1}(\mathbf{S})$. The Torelli group is torsion-free, and is trivial in the case of the sphere or torus.

\subsection{Graphs}

We use graph-theoretic terminology consistent with its use in [2]. We remind the reader of the less familiar terms, and give the graph-theoretic definitions of those terms that may be used in different ways in ordinary topology.

Throughout this work, $G$ will denote a connected, finite linear graph. We include the possibility that $G$ may contain loops or parallel edges. $E=E(G)$ will denote the edge set of $G$, and we use the symbols $a, b, c, e$ to denote edges of $G$. For $E^{\prime} \subset E(G), G-E^{\prime}$ denotes the subgraph obtained from $G$ by deleting the edges in $E^{\prime}$, while $G+E^{\prime \prime}$ is the graph obtained from $G$ by adding a set of edges $E^{\prime \prime}$. If $E=\{e\}$, then we write $G-e$ and $G+e$ instead of $G-\{e\}$ and $G+\{e\}$. A bond $E^{\prime}$ in $G$ is a minimal subset of $E(G)$ such that $G-E^{\prime}$ is disconnected. Note that $G-E^{\prime}$ consists of precisely two components. We say that the edge $e$ is a cut edge if $G-e$ is disconnected. We use the symbols $u, v, x, y$ to denote vertices of $G$. The degree of a vertex $v$ is the number of edges incident with $v$, each loop counting as two edges.

A $\left(v_{0}, v_{n}\right)-$ walk $W$ of length $n$ is a finite nonempty alternating sequence, $W=$ $v_{0} e_{1} v_{1} e_{2} v_{2} \ldots e_{n} v_{n}$, of vertices and edges such that the ends of the edge $e_{i}$ are the vertices $v_{i-1}$ and $v_{i}$ for $1 \leq i \leq n$. If the edges of $W$ are distinct, $W$ is called a trail. A cycle in $G$ is a closed trail of positive length whose origin and internal vertices are distinct. Thus a cycle is an embedded circle in $G$. For our purposes, to denote a trail or cycle, it will be enough to give its sequence of edges, and we do not distinguish between a closed trail $W$ and another closed trail whose sequence of edges is a cyclic permutation of $W$ 's.

A spanning tree $T$ is a subgraph of $G$ with the same vertex set as $G$ such that $T$ contains no cycles. The number of edges in any spanning tree is equal to one less than the number of vertices of $G$. Note that if $T$ is a spanning tree, and $e$ is an edge of $G$ not in $T$, then $T+e$ contains a unique cycle $C$, and $e$ is an edge of $C$, so the rank of $\pi_{1}(G)$ is equal to the number of edges of $G$ outside any spanning tree. Every connected graph contains a spanning tree. 


\section{Reduction Systems and Reduction System Graphs}

By a reduction system $\mathfrak{E}$ on $\mathbf{S}$ we mean a collection of simple closed curves on $\mathbf{S}$ that are homotopically nontrivial, pairwise disjoint, and pairwise nonisotopic. We use the symbols $\mathfrak{a}, \mathfrak{b}, \mathfrak{c}, \mathfrak{e}$ to denote the elements of a reduction system $\mathfrak{E}$, and $\mathbf{S}_{\mathfrak{E}}$ to denote the natural compactification of $\mathbf{S} \backslash \mathfrak{E}$; that is, "S cut along E."

We partition the set $\mathfrak{E}=\left\{\mathfrak{e}_{1}, \mathfrak{e}_{2}, \ldots, \mathfrak{e}_{n}\right\}$ according to the equivalence relation $\sim$ generated by the rule

$$
\mathfrak{e}_{i} \sim \mathfrak{e}_{j} \quad \text { if }\left\{\begin{array}{l}
\mathfrak{e}_{i}=\mathfrak{e}_{j} \\
\text { or } \\
\left\{\mathfrak{e}_{i}, \mathfrak{e}_{j}\right\} \text { is a minimal separating set in } \mathfrak{E} .
\end{array}\right.
$$

Here, " $\left\{\mathfrak{e}_{i}, \mathfrak{e}_{j}\right\}$ is a minimal separating set" means that $\mathbf{S}_{\left\{\mathfrak{e}_{i}, \mathfrak{e}_{j}\right\}}$ is disconnected, but both $\mathbf{S}_{\left\{\mathfrak{e}_{i}\right\}}$ and $\mathbf{S}_{\left\{\mathfrak{e}_{j}\right\}}$ are connected. There are three types of $\sim$-equivalence classes:

(i) Singleton classes $\left\{\mathfrak{a}_{1}\right\},\left\{\mathfrak{a}_{2}\right\}, \ldots,\left\{\mathfrak{a}_{p}\right\}$ consisting of the separating curves $\mathfrak{a}_{1}, \mathfrak{a}_{2}, \ldots, \mathfrak{a}_{p}$ in $\mathfrak{E}$. Such a curve wil be called an a-type curve.

(ii) Classes $\left\{\mathfrak{b}_{11}, \ldots, \mathfrak{b}_{1 q_{1}}\right\},\left\{\mathfrak{b}_{21}, \ldots, \mathfrak{b}_{2 q_{2}}\right\}, \ldots,\left\{\mathfrak{b}_{r 1}, \ldots, \mathfrak{b}_{r q_{r}}\right\}$ of cardinality at least 2. Each such class $\left\{\mathfrak{b}_{i 1}, \ldots, \mathfrak{b}_{i n_{i}}\right\}$ is characterized by the following three properties:

(a) No curve $\mathfrak{b}_{i j}$ is separating.

(b) $\mathfrak{b}_{i j}$ is homologous to $\mathfrak{b}_{i j^{\prime}}$ for every pair $\mathfrak{b}_{i j}, \mathfrak{b}_{i j^{\prime}}$.

(c) Maximal with respect to (a) and (b).

A curve in such a class will be called a b-type curve.

(iii) Singleton classes $\left\{\mathfrak{c}_{1}\right\},\left\{\mathfrak{c}_{2}\right\}, \ldots,\left\{\mathfrak{c}_{s}\right\}$ where each $\mathfrak{c}_{i}$ is non-separating and is homologous to no other curve in $\mathfrak{E}$. Such a curve will be called a $c$-type curve.

According to (i), (ii), and (iii) above, we write

$$
\mathfrak{E}=\left\{\mathfrak{a}_{1}, \ldots, \mathfrak{a}_{p}, \mathfrak{b}_{11}, \ldots, \mathfrak{b}_{1 q_{1}}, \ldots, \mathfrak{b}_{r 1}, \ldots, \mathfrak{b}_{r q_{r}}, \mathfrak{c}_{1}, \ldots, \mathfrak{c}_{s}\right\} .
$$

We use $\mathfrak{E}$ to define a graph $G_{\mathfrak{E}}$, which we call the reduction system graph of $\mathfrak{E}$, as follows:

- The vertices of $G_{\mathfrak{E}}$ correspond to the components of $\mathbf{S}_{\mathfrak{E}}$.

- The edges of $G_{\mathfrak{E}}$ correspond to the curves in the reduction system $\mathfrak{E}$, with: 
- (Links) Two distinct vertices are connected by the edge $e_{i}$ if and only if the curve $\mathfrak{e}_{i}$ in $\mathfrak{E}$ is a common boundary curve of the two components of $\mathbf{S}_{\mathfrak{E}}$ which correspond to the vertices in question.

- (Loops) A vertex has a loop $e_{i}$ if and only if the curve $\mathfrak{e}_{i}$ in $\mathfrak{E}$ represents two boundary curves of the component of $\mathbf{S}_{\mathfrak{E}}$ which corresponds to the vertex in question.

Note that $G_{\mathfrak{E}}$ is connected, and that any connected graph $G$ is $G_{\mathfrak{E}}$ for some surface $\mathbf{S}$ and some reduction system $\mathfrak{E}$ on $\mathbf{S}$. However, the genus of $\mathbf{S}$ is not determined by $G$, any two possible $\mathbf{S}$ 's differing by the genera of their complementary components. But, unless $G$ is the graph consisting of a single vertex and either no edges or a single loop, then $\operatorname{genus}(\mathbf{S}) \geq \operatorname{rank}\left(\pi_{1}(G)\right)+$ (number of vertices of degree $\leq 2$ ).

Since $\mathbf{S}$ and $\mathfrak{E}$ will be fixed, we will denote $G_{\mathfrak{E}}$ simply by $G$.

The $\sim$-equivalence relation on the curves in $\mathfrak{E}$ induces a $\sim$-equivalence relation on the edge set $E(G)=\left\{e_{1}, e_{2}, \ldots, e_{n}\right\}$ of $G$. It is generated by

$$
e_{i} \sim e_{j} \quad \text { if }\left\{\begin{array}{l}
e_{i}=e_{j} \\
\text { or } \\
\left\{e_{i}, e_{j}\right\} \text { is a bond. }
\end{array}\right.
$$

(Again, it should be noted that this equivalence relation may be defined for any graph $G$.) The three types of equivalence classes described above become, for $G$,

(i) Singleton classes $\left\{a_{1}\right\}, \ldots,\left\{a_{p}\right\}$ consisting of the cut edges $a_{1}, \ldots, a_{p}$ of $G$. Such an edge will be called an a-type edge.

(ii) Classes $\left\{b_{11}, \ldots, b_{1 q_{1}}\right\},\left\{b_{21}, \ldots, b_{2 q_{2}}\right\}, \ldots,\left\{b_{r 1}, \ldots, b_{r q_{r}}\right\}$ of cardinality at least 2. Each such class is characterized by the following three properties:

(a) No edge $b_{i j}$ is a cut edge.

(b) $\left\{b_{i j}, b_{i j^{\prime}}\right\}$ is a bond for every pair $b_{i j}, b_{i j^{\prime}}$.

(c) Maximal with respect to (a) and (b).

An edge in such a class will be called a b-type edge.

(iii) Singleton classes $\left\{c_{1}\right\}, \ldots,\left\{c_{s}\right\}$ where each $c_{i}$ is not a cut edge, and forms a 2 -edge bond with no other edge of $G$. Such an edge will be called a c-type edge.

According to (i), (ii), and (iii) above, we write

$$
E(G)=\left\{a_{1}, \ldots, a_{p}, b_{11}, \ldots, b_{1 q_{1}}, b_{21}, \ldots, b_{2 q_{2}}, \ldots, b_{r 1}, \ldots, b_{r q_{r}}, c_{1}, \ldots, c_{s}\right\} .
$$



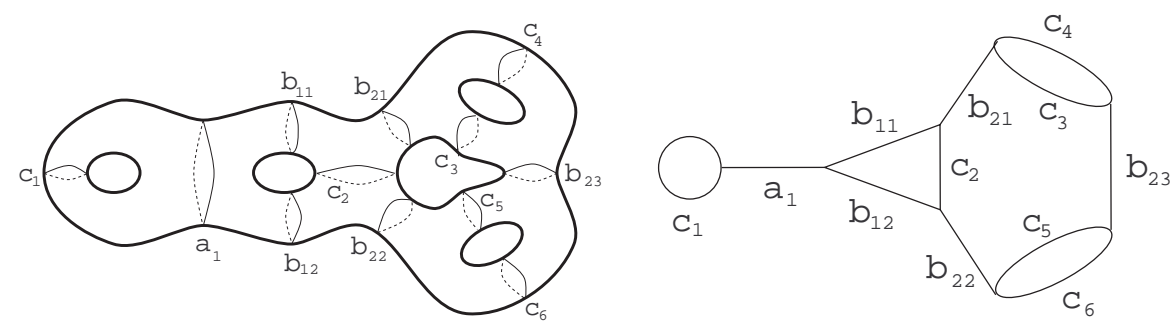

A typical example is shown above.

Now let $\mathfrak{h}$ be a simple closed curve on $\mathbf{S}$ that intersects each element of $\mathfrak{E}$ transversely at most once. Starting at any point on $\mathfrak{h}$ and travelling in either direction gives a cyclic ordering of the reduction curves which $\mathfrak{h}$ intersects, thus defining a closed trail $H$ in $G$. Note that $H$ is a cycle in $G$ if and only if $\mathfrak{h} \cap \mathbf{S}_{i}$ is either empty or is a single (that is, connected) arc, for every component $\mathbf{S}_{i}$ of $\mathbf{S}_{\mathfrak{E}}$. Likewise, given a closed trail $H$ in $G$, there is such a curve $\mathfrak{h}$ on $\mathbf{S}$ defining $H$. The fact that the isotopy class of $\mathfrak{h}$ is never unique is not important for our purposes.

The following figure shows a typical example. Note that $\mathfrak{h}_{1}$ and $\mathfrak{h}_{2}$ are nonisotopic curves which both define the cycle $H=b_{11} c_{2} b_{12}$.
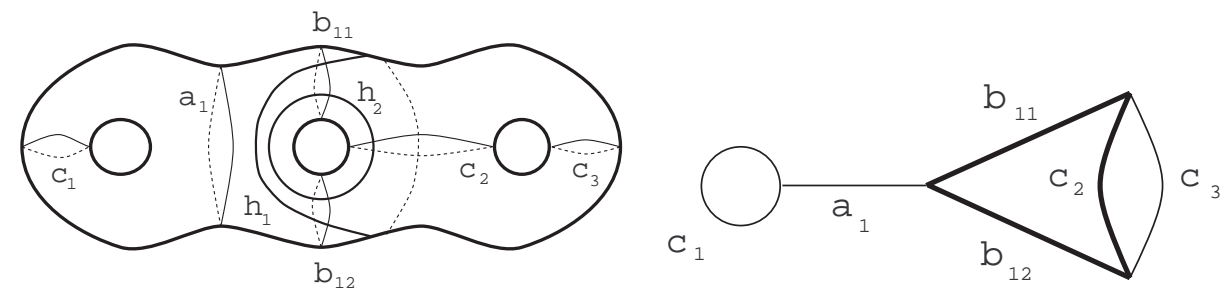

The remainder of this section presents some purely graph-theoretic results, concluding with Theorem 2.1, which is used in the following section. So for the remainder of this section, let $G$ denote an arbitrary connected graph. We explain here the notation and terminology we use. Given a subgraph $H$ of $G$, we let $G \bullet H$ denote the graph obtained by deleting every edge $e$ of $H$ and identifying the ends of $e$. Equivalently, thinking of $G$ as a CW-complex and $H$ as a subcomplex, $G \bullet H$ is the complex obtained from $G$ by crushing each component of $H$ to a point. Thus, we have a quotient ("contraction") map $p: G \rightarrow G \bullet H$. Next, by a cut vertex of $G$, we mean a vertex $v$ of $G$ such that when $v$, and only $v$, is removed from the topological space $G$, the resulting 
space is disconnected. (This is not the definition used by graph theorists, but is an equivalent topological one.) A block is a connected graph without cut vertices, and a block of a graph is a subgraph that is a block and is maximal with repsect to that property. Any graph is the union of its blocks. We leave the proofs of the first two lemmas to the reader.

Lemma 2.1 If $G$ has no cut edges, then any two vertices of $G$ are connected by two edge-disjoint paths.

Lemma 2.2 Let $b_{1}$ and $b_{2}$ be edges of $G$ such that $\left\{b_{1}, b_{2}\right\}$ is a bond. If $C$ is a cycle in $G$, and $b_{1}$ is an edge of $C$, then so is $b_{2}$.

Lemma 2.3 Let $c$ be a c-type edge in $G$ that is not a loop. Then $c$ is contained in two cycles, the intersection of whose edge sets is precisely $c$.

Proof Assume that $G$ is a block. If $G$ has exactly two vertices, then each edge of $G$ is a link, and $G$ must have at least three edges, since $c$ is a c-type edge. The result is clear in this case. Otherwise, $G$ has at least three vertices and no cut edges. Consider the graph $G-c$. If $G-c$ has a cut edge $e$, then $G-\{c, e\}$ is not connected, so $\{c, e\}$ is a bond of $G$. This contradicts the fact that $c$ is a c-type edge. So $G-c$ has no cut edges. By Lemma 2.1, there are two edge-disjoint paths $P$ and $P^{\prime}$ in $G-c$ connecting the ends of $c$. Then the cycles $C=P+c$ and $C^{\prime}=P^{\prime}+c$ have exactly the edge $c$ in common. In the case that $G$ is not a block, we let $B$ be the block of $G$ containing $c$. It is easy to see that $c$ is a c-type edge of $B$, so we apply the first case to $B$ and find two such cycles within $B$.

Theorem 2.1 Let $G$ have edge set

$$
E(G)=\left\{a_{1}, \ldots, a_{p}, b_{11}, \ldots, b_{1 q_{1}}, \ldots, b_{r 1}, \ldots, b_{r q_{r}}, c_{1}, \ldots, c_{s}\right\},
$$

notated according to a-, $\mathrm{b}-$, and c-type equivalence classes. Let $w: E(G) \rightarrow$ $\mathbb{Z}$ be a weighting of $G$. Then $w(H)=0$ for every cycle $H$ in $G$ if and only if

(i) $w\left(c_{i}\right)=0,1 \leq i \leq s$, and

(ii) $w\left(b_{j 1}\right)+w\left(b_{j 2}\right)+\cdots+w\left(b_{j q_{j}}\right)=0,1 \leq j \leq r$.

Proof $\Rightarrow$ Assume that $w(H)=0$ for every cycle $H$ in $G$. 
(i) Let $c$ be a c-type edge with ends $u$ and $v$. If $c$ is a loop, then $w(c)=0$, by hypothesis. Otherwise, there are two edge-disjoint $(u, v)$-paths, $P$ and $\overline{P^{\prime}}$, in $G-c$. We have three cycles: $P+c, P^{\prime}+c$, and $P+P^{\prime}$. Thus:

$$
\left.\begin{array}{r}
w(P)+w(c)=w(P+c)=0 \\
w\left(P^{\prime}\right)+w(c)=w\left(P^{\prime}+c\right)=0 \\
w(P)+w\left(P^{\prime}\right)=w\left(P+P^{\prime}\right)=0
\end{array}\right\} \Longrightarrow w(c)=0
$$

(ii) Let $B$ be the equivalence class of the b-type edge $b$, and $\bar{B}=B \backslash\{b\}$. Let $p: G \rightarrow G \bullet \bar{B}$ be the contraction map. Suppose that $b$ is a cut edge of $G \bullet \bar{B}$, separating it into two components $G_{1}$ and $G_{2}$. Then the restriction of $p$ to $G-b$ maps onto the disconnected space $G_{1} \cup G_{2}$, and so $G-b$ is disconnected. This is a contradiction to the hypothesis that $b$ is a b-type edge of $G$. We obtain a similar contradiction if we suppose $\{b, e\}$ is a bond in $G \bullet \bar{B}$. Thus $b$ is a c-type edge in $G \bullet \bar{B}$. If $b$ is a loop in $G \bullet \bar{B}$, then $p^{-1}(b)=B$, which therefore forms a cycle in $G$. So equation (ii) holds for the equivalence class of $b$.

If $b$ is not a loop in $G \bullet \bar{B}$, then by Lemma 2.3 there are two cycles $\bar{H}$ and $\overline{H^{\prime}}$ in $G \bullet \bar{B}$, the intersection of whose edge sets is $\{b\}$. Lemma 2.2 implies that $p^{-1}(\bar{H})$ and $p^{-1}\left(\overline{H^{\prime}}\right)$ are cycles $H$ and $H^{\prime}$, respectively, the intersection of whose edge sets is precisely $B$. Thus we have

$$
\left.\begin{array}{r}
0=w(H)=w(B)+w(H-B) \\
0=w\left(H^{\prime}\right)=w(B)+w\left(H^{\prime}-B\right)
\end{array}\right\} \Longrightarrow 0=2 w(B)+w\left(H \Delta H^{\prime}\right)=2 w(B) .
$$

And so, $w(B)=0$. Here we have used the fact that the symmetric difference $H \Delta H^{\prime}$ of the cycles $H$ and $H^{\prime}$ is a disjoint union of cycles (regarded as sets of edges).

$\Leftarrow$ Assume that

(i) $w\left(c_{i}\right)=0,1 \leq i \leq s$, and

(ii) $w\left(b_{j 1}\right)+w\left(b_{j 2}\right)+\cdots+w\left(b_{j q_{j}}\right)=0,1 \leq j \leq r$.

Let $H$ be a cycle in $G$. $H$ contains no a-type edges, since they are cut edges, and by Lemma 2.2, if $H$ contains one edge of a b-type class, then it contains the whole class. So the assumptions imply that $w(H)=0$.

\section{Abelian Subgroups in the Torelli Group}

We at first consider a specific type of Abelian subgroup of the Torelli group $\mathcal{T}(\mathbf{S})$, namely one consisting of multitwists - that is, compositions of left and right Dehn twists about a fixed reduction system on $\mathbf{S}$. 
Theorem 3.1 Let $\mathbf{S}$ be a closed, connected, oriented surface, and let

$$
\mathfrak{E}=\left\{\mathfrak{a}_{1}, \ldots, \mathfrak{a}_{p}, \mathfrak{b}_{11}, \ldots, \mathfrak{b}_{1 q_{1}}, \ldots, \mathfrak{b}_{r 1}, \ldots, \mathfrak{b}_{r q_{r}}, \mathfrak{c}_{1}, \ldots, \mathfrak{c}_{s}\right\}
$$

be a reduction system on $\mathbf{S}$, notated by $\mathrm{a}-, \mathrm{b}-$, and $\mathrm{c}$-type $\sim$-equivalence classes as in section 2. Let $\mathcal{D}_{\mathfrak{E}}$ be the multitwist group on $\mathfrak{E}$, and let

$$
f=D_{\mathfrak{a}_{1}}^{\alpha_{1}} \cdots D_{\mathfrak{a}_{p}}^{\alpha_{p}} D_{\mathfrak{b}_{11}}^{\beta_{11}} \cdots D_{\mathfrak{b}_{1 q_{1}}}^{\beta_{1 q_{1}}} \cdots D_{\mathfrak{b}_{r 1}}^{\beta_{r 1}} \cdots D_{\mathfrak{b}_{r q_{r}}}^{\beta_{r q_{r}}} D_{\mathfrak{c}_{1}}^{\gamma_{1}} \cdots D_{\mathfrak{c}_{s}}^{\gamma_{s}}
$$

be an element of $\mathcal{D}_{\mathfrak{E}}$. Then $f$ is an element of $\mathcal{D}_{\mathfrak{E}} \cap \mathcal{T} \equiv \mathcal{T}_{\mathfrak{E}}$, which we call the Torelli multitwist group of $\mathfrak{E}$, if and only if

(i) $\gamma_{i}=0,1 \leq i \leq s$, and

(ii) $\beta_{j 1}+\beta_{j 2}+\cdots+\beta_{j q_{j}}=0,1 \leq j \leq r$.

Consequently, $\mathcal{T}_{\mathfrak{E}}$ is a free Abelian group of rank

$$
p+\left(q_{1}-1\right)+\left(q_{2}-1\right)+\cdots+\left(q_{r}-1\right)=p+q_{1}+q_{2}+\cdots+q_{r}-r .
$$

Remark A set of $\sim$-equivalence class representatives of the curves in $\mathfrak{E}$ is in general not linearly independent in $H_{1}(\mathbf{S})$, so the nondegeneracy of the algebraic intersection $\langle$,$\rangle is not sufficient to prove the theorem.$

Proof $\Rightarrow$ Assume that $f \in \mathcal{T}_{\mathfrak{E}}$.

Let $G$ be the reduction system graph of $\mathfrak{E}$ with edge set $E(G)$. We weight each edge of $G$ according to the exponent in $f$ of the twist about its corresponding curve in $\mathfrak{E}$, giving $w: E(G) \rightarrow \mathbb{Z}$.

Let $H=e_{1} e_{2}, \ldots, e_{n}$ be a cycle in $G$. Then, as in section $2, H$ is defined by any simple closed curve $\mathfrak{h}$ on $\mathbf{S}$ that intersects each of the corresponding curves $\mathfrak{e}_{1}, \mathfrak{e}_{2}, \ldots, \mathfrak{e}_{n}$ of $\mathfrak{E}$ exactly once, and does not intersect any of the other curves of $\mathfrak{E}$. Orient $\mathfrak{h}$. Then orient the curves $\mathfrak{e}_{1}, \mathfrak{e}_{2}, \ldots, \mathfrak{e}_{n}$ so that $\left\langle\mathfrak{e}_{i}, \mathfrak{h}\right\rangle=1$. So we have

$$
0=\langle\mathfrak{h}, \mathfrak{h}\rangle=\langle\mathfrak{h}, f(\mathfrak{h})\rangle=\left\langle\mathfrak{h}, \mathfrak{h}+\epsilon_{1} \mathfrak{e}_{1}+\epsilon_{2} \mathfrak{e}_{2}+\cdots+\epsilon_{n} \mathfrak{e}_{n}\right\rangle=\epsilon_{1}+\epsilon_{2}+\cdots+\epsilon_{n},
$$

where $\epsilon_{i}=w\left(e_{i}\right)$. Hence the weight of every cycle in $G$ is zero. The conclusion follows from Theorem 2.1.

$\Leftarrow$ Assume that

(i) $\gamma_{i}=0,1 \leq i \leq s$, and

(ii) $\beta_{j 1}+\beta_{j 2}+\cdots+\beta_{j q_{j}}=0,1 \leq j \leq r$. 
Since $H_{1}(\mathbf{S})$ has a basis consisting of simple closed curves, in order to prove that $f \in \mathcal{T}$, it suffices to show that in $H_{1}(\mathbf{S})$, we have $f(\mathfrak{h})=\mathfrak{h}$ for any simple closed curve $\mathfrak{h}$ on $\mathbf{S}$. Note that for any such $\mathfrak{h}$, we have $\left\langle\mathfrak{a}_{i}, \mathfrak{h}\right\rangle=0,1 \leq i \leq p$, and after orienting $\mathfrak{h}$ and then each $\mathfrak{b}_{i j}$ so that $\left\langle\mathfrak{b}_{i j}, \mathfrak{h}\right\rangle=\left\langle\mathfrak{b}_{i 1}, \mathfrak{h}\right\rangle$, we have $\mathfrak{b}_{i j}=\mathfrak{b}_{i 1}, 2 \leq j \leq q_{i}, 1 \leq i \leq r$. Let $\delta_{i}=\left\langle\mathfrak{b}_{i 1}, \mathfrak{h}\right\rangle$. Then in $H_{1}(\mathbf{S})$ we have:

$$
\begin{aligned}
f(\mathfrak{h})= & D_{\mathfrak{a}_{1}}^{\alpha_{1}} \cdots D_{\mathfrak{a}_{p}}^{\alpha_{p}} D_{\mathfrak{b}_{11}}^{\beta_{11}} \cdots D_{\mathfrak{b}_{1 q_{1}}}^{\beta_{1 q_{1}}} \cdots D_{\mathfrak{b}_{r 1}}^{\beta_{r 1}} \cdots D_{\mathfrak{b}_{r q_{r}}}^{\beta_{r q_{r}}} D_{\mathfrak{c}_{1}}^{\gamma_{1}} \cdots D_{\mathfrak{c}_{s}}^{\gamma_{s}}(\mathfrak{h}) \\
= & \mathfrak{h}+\beta_{11}\left\langle\mathfrak{b}_{11}, \mathfrak{h}\right\rangle \mathfrak{b}_{11}+\cdots+\beta_{1 q_{1}}\left\langle\mathfrak{b}_{1 q_{1}}, \mathfrak{h}\right\rangle \mathfrak{b}_{1 q_{1}}+\cdots \\
& \quad+\beta_{r 1}\left\langle\mathfrak{b}_{r 1}, \mathfrak{h}\right\rangle \mathfrak{b}_{r 1}+\cdots+\beta_{r q_{r}}\left\langle\mathfrak{b}_{r q_{r}}, \mathfrak{h}\right\rangle \mathfrak{b}_{r q_{r}} \\
= & \mathfrak{h}+\delta_{1}\left(\beta_{11}+\cdots+\beta_{1 q_{1}}\right) \mathfrak{b}_{11}+\cdots+\delta_{r}\left(\beta_{r 1}+\cdots+\beta_{r q_{r}}\right) \mathfrak{b}_{r 1} \\
= & \mathfrak{h}
\end{aligned}
$$

Theorem 3.2 Let $\mathbf{S}$ be a closed connected oriented surface, and let $\mathfrak{E}=$ $\left\{\mathfrak{e}_{1}, \mathfrak{e}_{2}, \ldots, \mathfrak{e}_{n}\right\}$ be a reduction system on $\mathbf{S}$. Let $f=D_{\mathfrak{e}_{1}}^{\epsilon_{1}} D_{\mathfrak{e}_{2}}^{\epsilon_{2}} \cdots D_{\mathfrak{e}_{n}}^{\epsilon_{n}}$ be a multitwist on $\mathfrak{E}$. Let $G$ be the reduction system graph of $\mathfrak{E}$, and define a weighting $w: E(G) \rightarrow \mathbb{Z}$ of $G$ by $w\left(e_{i}\right)=\epsilon_{i}$. Then $f$ is in the Torelli multitwist group $\mathcal{T}_{\mathfrak{E}}$ if and only if the weight of every cycle in $G$ is zero.

Proof Partition $\mathfrak{E}$ into $\sim$-equivalence classes, so

$$
\mathfrak{E}=\left\{\mathfrak{a}_{1}, \ldots, \mathfrak{a}_{p}, \mathfrak{b}_{11}, \ldots, \mathfrak{b}_{1 q_{1}}, \ldots, \mathfrak{b}_{r 1}, \ldots, \mathfrak{b}_{r q_{r}}, \mathfrak{c}_{1}, \ldots, \mathfrak{c}_{s}\right\} .
$$

Theorems 2.1 and 3.1 show the conditions to be equivalent.

Given a pair, $\mathfrak{e}_{1}$ and $\mathfrak{e}_{2}$, of disjoint, non-separating, but homologous simple closed curves on $\mathbf{S}$, we call $D_{\mathfrak{e}_{1}} D_{\mathfrak{e}_{2}}^{-1}$ a bounding-pair map or BP map. Powell [5] has shown that the Torelli group $\mathcal{T}$ is generated by BP maps and Dehn twists about separating simple closed curves.

Corollary 3.1 Let $\mathbf{S}$, E, $\mathcal{D}_{\mathfrak{E}}$, and $\mathcal{T}_{\mathfrak{E}}$ be as in Theorem 3.1. Let $\mathcal{D}^{\prime}$ be the subgroup of $\mathcal{M}(\mathbf{S})$ generated by

(i) BP maps about bounding pairs in $\mathfrak{E}$, and

(ii) Dehn twists about separating curves in $\mathfrak{E}$.

Then $\mathcal{D}^{\prime}=\mathcal{D}_{\mathfrak{E}} \cap \mathcal{T}=\mathcal{T}_{\mathfrak{E}}$.

Proof By the definition of $\mathcal{D}_{\mathfrak{E}}$, it is clear that every generator of $\mathcal{D}^{\prime}$ is in $\mathcal{D}_{\mathfrak{E}}$. By Powell's result noted above, every generator of $\mathcal{D}^{\prime}$ is in $\mathcal{T}$. Thus $\mathcal{D}^{\prime} \subseteq \mathcal{D}_{\mathfrak{E}} \cap \mathcal{T}$. We must show that $\mathcal{D}_{\mathfrak{E}} \cap \mathcal{T} \subseteq \mathcal{D}^{\prime}$ 
Let $f \in \mathcal{D}_{\mathfrak{E}} \cap \mathcal{T}$. By Theorem 3.1, we know that

$$
f=D_{\mathfrak{a}_{1}}^{\alpha_{1}} \cdots D_{\mathfrak{a}_{p}}^{\alpha_{p}} D_{\mathfrak{b}_{11}}^{\beta_{11}} \cdots D_{\mathfrak{b}_{1 q_{1}}}^{\beta_{1 q_{1}}} D_{\mathfrak{b}_{21}}^{\beta_{21}} \cdots D_{\mathfrak{b}_{2 q_{2}}}^{\beta_{2 q_{2}}} \cdots D_{\mathfrak{b}_{r 1}}^{\beta_{r 1}} \cdots D_{\mathfrak{b}_{r q_{r}}}^{\beta_{r q_{r}}},
$$

where $\beta_{i 1}+\beta_{i 2}+\cdots+\beta_{i q_{i}}=0, \quad 1 \leq i \leq r$. Since each $D_{\mathfrak{a}_{i}}^{\alpha_{i}}$ is a product of type-(ii) generators of $\mathcal{D}^{\prime}$, we will be done if we write $D_{\mathfrak{b}_{i 1}}^{\beta_{i 1}} D_{\mathfrak{b}_{i 2}}^{\beta_{i 2}} \cdots D_{\mathfrak{b}_{i q_{i}}}^{\beta_{i q_{i}}}$ as a product of BP maps. We do this:

$$
D_{\mathfrak{b}_{i 1}}^{\beta_{i 1}} D_{\mathfrak{b}_{i 2}}^{\beta_{i 2}} \cdots D_{\mathfrak{b}_{i q_{i}}}^{\beta_{i q_{i}}}=\left(D_{\mathfrak{b}_{i 2}} D_{\mathfrak{b}_{i 1}}^{-1}\right)^{\beta_{i 2}}\left(D_{\mathfrak{b}_{i 3}} D_{\mathfrak{b}_{i 1}}^{-1}\right)^{\beta_{i 3}} \cdots\left(D_{\mathfrak{b}_{i q_{1}}} D_{\mathfrak{b}_{i 1}}^{-1}\right)^{\beta_{i q_{i}}},
$$

where we note that $-\beta_{i 2}-\beta_{i 3}-\cdots-\beta_{i q_{i}}=\beta_{i 1}$.

Corollary 3.2 Let $\mathbf{S}$ be a closed, connected, oriented surface, and let

$$
\mathfrak{E}=\left\{\mathfrak{a}_{1}, \ldots, \mathfrak{a}_{p}, \mathfrak{b}_{11}, \ldots, \mathfrak{b}_{1 q_{1}}, \ldots, \mathfrak{b}_{r 1}, \ldots, \mathfrak{b}_{r q_{r}}, \mathfrak{c}_{1}, \ldots, \mathfrak{c}_{s}\right\}
$$

be a reduction system on $\mathbf{S}$, notated by $\mathrm{a}-, \mathrm{b}-$, and c-type $\sim$-equivalence classes as in section 2. Let $\mathcal{D}_{\mathfrak{E}}$ be the multitwist group on $\mathfrak{E}$, and let

$$
f=D_{\mathfrak{a}_{1}}^{\alpha_{1}} \cdots D_{\mathfrak{a}_{p}}^{\alpha_{p}} D_{\mathfrak{b}_{11}}^{\beta_{11}} \cdots D_{\mathfrak{b}_{1 q_{1}}}^{\beta_{1 q_{1}}} \cdots D_{\mathfrak{b}_{r 1}}^{\beta_{r 1}} \cdots D_{\mathfrak{b}_{r q_{r}}}^{\beta_{r q_{r}}} D_{\mathfrak{c}_{1}}^{\gamma_{1}} \cdots D_{\mathfrak{c}_{s}}^{\gamma_{s}}
$$

be an element of $\mathcal{D}_{\mathfrak{E}}$. Let $m \geq 2$ be an integer.

Then $f \in \Gamma_{\mathbf{S}}(m) \equiv\left\{g \in \mathcal{M}(\mathbf{S}): g\right.$ acts trivially on $\left.H_{1}\left(\mathbf{S} ; \mathbb{Z}_{m}\right)\right\}$ if and only if

(i) $\gamma_{i} \equiv 0(\bmod m), 1 \leq i \leq s$, and

(ii) $\beta_{j 1}+\beta_{j 2}+\cdots+\beta_{j q_{j}} \equiv 0(\bmod m), 1 \leq j \leq r$.

Let $\mathbf{S}$ be the surface of genus $g \geq 2$ and $\mathfrak{E}$ the reduction system on $\mathbf{S}$ shown below. Since $\mathfrak{E}$ consists of $2 g-3$ a-type curves, $\operatorname{rank}\left(\mathcal{T}_{\mathfrak{E}}\right)=2 g-3$. This example, along with Theorem 4.1 below, shows that the maximal rank of an Abelian subgroup of the Torelli group is attained by a multitwist group.

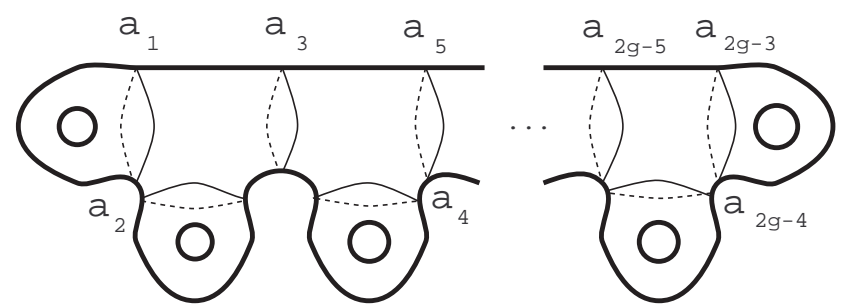

Algebraic 85 Geometric Topology, Volume 2 (2002) 
Remark One particular naively-expected symplectic analogue of Theorem 3.1 is not true:

"Conjecture" Let $(\mathcal{V},\langle\rangle$,$) be a symplectic lattice of rank 2 g$, where $g \geq$ 2 . Let $\left\{v_{1}, v_{2}, \ldots, v_{n}\right\}$ be a set of primitive vectors in $\mathcal{V}$ that are pairwise linearly independent and symplectically orthogonal. Let $T_{i}$ be the transvection corresponding to the vector $v_{i}$. Thus $T_{i}(w)=w+\left\langle v_{i}, w\right\rangle$ for any $w \in \mathcal{V}$. Let $m_{1}, m_{2}, \ldots, m_{n}$ be integers. Then the "multitransvection" $T=T_{1}^{m_{1}} T_{2}^{m_{2}} \cdots T_{n}^{m_{n}}$ is the identity on $\mathcal{V}$ if and only if $m_{i}=0,1 \leq i \leq n$.

But now let $\left\{a_{1}, b_{1}, a_{2}, b_{2}, \ldots, a_{g}, b_{g}\right\}$ be the standard symplectic basis for $\mathcal{V}$, and for $i=1,2,3$, and 4 , let $v_{i}=a_{1}+i a_{2}$. Let $m_{1}=1, m_{2}=-3, m_{3}=3$, and $m_{4}=-1$. One can verify that $T=T_{1}^{m_{1}} T_{2}^{m_{2}} T_{3}^{m_{3}} T_{4}^{m_{4}}=i d_{\mathcal{V}}$. This shows the conjecture to be false.

Now we prove that for any closed oriented surface of genus $g \geq 2$, the general Abelian subgoup of its Torelli group has rank $\leq 2 g-3$. We first give two lemmas.

Lemma 3.1 Let $\mathbf{S}$ be a closed, connected, oriented surface, and $\mathfrak{E}$ a reduction system on $\mathbf{S}$ with reduction system graph $G$. Let $\mathcal{T}_{\mathfrak{E}}$ be the Torelli multitwist group on $\mathfrak{E}$, as in Theorem 3.1. Then $\operatorname{rank}\left(\mathcal{T}_{\mathfrak{E}}\right) \leq \nu-1$, where $\nu$ is the number of vertices of $G$, or, equivalently, the number of components of $\mathbf{S}_{\mathfrak{E}}$.

Proof Let $G$ have edge setl

$$
E(G)=\left\{a_{1}, \ldots, a_{p}, b_{11}, \ldots, b_{1 q_{1}}, b_{21}, \ldots, b_{2 q_{2}}, \ldots, b_{r 1}, \ldots, b_{r q_{r}}, c_{1}, \ldots, c_{s}\right\} .
$$

Let $E^{\prime}=\left\{b_{11}, \ldots, b_{1\left(q_{1}-1\right)}, b_{21}, \ldots, b_{2\left(q_{2}-1\right)}, \ldots, b_{r 1}, \ldots, b_{r\left(q_{r}-1\right)}\right\} \subseteq E(G)$, and let $G^{\prime}=G\left[E^{\prime}\right]$. Then $G^{\prime}$ contains no cycles, since any cycle containing one edge of a b-type class contains the whole class. Therefore, $G^{\prime}$ is contained in a spanning tree $T$ of $G$. Since each $a_{i}$ is a cut edge, $T$ contains $a_{i}, 1 \leq i \leq p$.

So $T$ contains the set of edges $E^{\prime} \cup\left\{a_{1}, a_{2}, \ldots, a_{p}\right\}$. But by Theorem 3.1, the cardinality of this set is equal to the rank of $\mathcal{T}_{\mathfrak{E}}$. This gives us

$$
\nu-1=\operatorname{card}(E(T)) \geq p+\left(q_{1}-1\right)+\cdots+\left(q_{r}-1\right)=\operatorname{rank}\left(\mathcal{T}_{\mathfrak{E}}\right) .
$$

Lemma 3.2 Let $\mathbf{S}$ be a closed, connected, oriented surface of genus $g \geq 2$, and let $\mathfrak{E}$ be a reduction system on $\mathbf{S}$. Let $\Omega$ denote the number of components of $\mathbf{S}_{\mathfrak{E}}$ not homeomorphic to a pair of pants or a one-holed torus. Let $\mathcal{T}_{\mathfrak{E}}$ be the Torelli multitwist group on $\mathfrak{E}$. Then $\operatorname{rank}\left(\mathcal{T}_{\mathfrak{E}}\right)+\Omega \leq 2 g-3$. 
Proof Let $G$ be the reduction system graph of $\mathfrak{E}$. We use the following notation:

- $\Gamma$ is the maximum genus of any component of $\mathbf{S}_{\mathfrak{E}}$.

- $\Delta$ is the maximum degree of any vertex of $G$, or, equivalently, the maximum number of boundary curves of any component of $\mathbf{S}_{\mathfrak{E}}$.

- $\nu_{b}$ is the number of vertices of $G$ of degree $b$, or, equivalently, the number of components of $\mathbf{S}_{\mathfrak{E}}$ with $b$ boundary curves.

- $\nu_{b}^{\gamma}\left(\nu_{b}^{\geq \gamma}\right)$ is the number of components of $\mathbf{S}_{\mathfrak{E}}$ of genus $\gamma(\geq \gamma)$ having $b$ boundary curves, or, equivalently, the number of vertices of $G$ of degree $b$ corresponding to a component of $\mathbf{S}_{\mathfrak{E}}$ of genus $\gamma(\geq \gamma)$.

So we have:

$$
\nu_{b}=\sum_{\gamma=0}^{\Gamma} \nu_{b}^{\gamma} \quad \text { and } \quad \nu=\sum_{b=1}^{\Delta} \nu_{b}
$$

But the assumption that each element of $\mathfrak{E}$ is homotopically nontrivial means $\nu_{1}^{0}=0$, and the assumption that the elements of $\mathfrak{E}$ are pairwise nonisotopic means $\nu_{2}^{0}=0$. So, in fact, $\nu=\nu_{1}^{\geq 1}+\nu_{2}^{\geq 1}+\nu_{3}+\nu_{4}+\cdots+\nu_{\Delta}$. Now, $\nu_{1}^{1}$ is the number of one-holed tori, and $\nu_{3}^{0}$ is the number of pairs of pants, so by the definition of $\Omega$, we have $\Omega=\nu_{1}^{\geq 2}+\nu_{2}^{\geq 1}+\nu_{3}^{\geq 1}+\nu_{4}+\cdots+\nu_{\Delta}$. Hence

$$
\begin{aligned}
2 g-2 & =-\chi(\mathbf{S}) \\
& =\sum_{\text {components } \mathbf{V} \text { of } \mathbf{S}_{\mathfrak{E}}}-\chi(\mathbf{V}) \\
& =\sum_{\gamma=1}^{\Gamma}(2 \gamma-1) \nu_{1}^{\gamma}+\sum_{\gamma=1}^{\Gamma}(2 \gamma) \nu_{2}^{\gamma}+\sum_{b=3}^{\Delta} \sum_{\gamma=0}^{\Gamma}(2 \gamma+b-2) \nu_{b}^{\gamma}
\end{aligned}
$$

By Lemma 3.1, $\operatorname{rank}\left(\mathcal{T}_{\mathfrak{E}}\right) \leq \nu-1$, so we have

$$
\begin{aligned}
\operatorname{rank}\left(\mathcal{T}_{\mathfrak{E}}\right) & +\Omega \leq \nu+\Omega-1 \\
& =\left(\nu_{1}^{\geq 1}+\nu_{1}^{\geq 2}+\cdots+\nu_{\Delta}\right)+\left(\nu_{1}^{\geq 2}+\nu_{2}^{\geq 1}+\nu_{3}^{\geq 1}+\nu_{4}+\cdots+\nu_{\Delta}\right)-1 \\
& =\left[\left(\nu_{1}^{1}+2 \nu_{1}^{\geq 2}\right)+2 \nu_{2}^{\geq 1}+\left(\nu_{3}^{0}+2 \nu_{3}^{\geq 1}\right)+2 \nu_{4}+2 \nu_{5}+\cdots+2 \nu_{\Delta}\right]-1 \\
& \leq\left[\sum_{\gamma=1}^{\Gamma}(2 \gamma-1) \nu_{1}^{\gamma}+\sum_{\gamma=1}^{\Gamma}(2 \gamma) \nu_{2}^{\gamma}+\sum_{b=3}^{\Delta} \sum_{\gamma=0}^{\Gamma}(2 \gamma+b-2) \nu_{b}^{\gamma}\right]-1 \\
& =-\chi(\mathbf{S})-1 \\
& =2 g-3
\end{aligned}
$$


Theorem 3.3 Let $\mathbf{S}$ be a closed, connected, oriented surface of genus $g \geq$ 2 , and let $\mathcal{A}$ be an Abelian subgroup of $\mathcal{T}$, the Torelli group of $\mathbf{S}$. Then $\operatorname{rank}(\mathcal{A}) \leq 2 g-3$.

Proof This proof is an adaptation of a analogous proof in [1]. That paper also introduces the reduction homomorphism and essential reduction system which we refer to here.

Let $f \in \mathcal{A}, f \neq 0$. By Thurston's classification, $f$ is either reducible, pseudoAnosov, or of finite order. Since $\mathcal{T}$ is torsion-free, $f$ cannot be of finite order. We consider the other two possibilities.

Case $1 f$ is pseudo-Anosov.

Let $\langle f\rangle$ denote the cyclic subgroup of $\mathcal{A}$ generated by $f$, and let $\mathcal{C}=C_{\mathcal{M}(\mathbf{S})}(\langle f\rangle)$, the centralizer of $\langle f\rangle$ in $\mathcal{M}(\mathbf{S})$. Then $\mathcal{A} \subseteq \mathcal{C}$ and $\mathcal{A}$ is torsion-free. We conclude by a theorem of McCarthy ([4], Corollary 3) that $\mathcal{A}$ is infinite cyclic. Hence $\operatorname{rank}(\mathcal{A})=1 \leq 2 g-3$.

Case $2 f$ is reducible.

Given $h \in \mathcal{A}$, let $\mathfrak{E}_{h}$ denote the essential reduction system of $h$, and let

$$
\mathfrak{E}=\bigcup_{h \in \mathcal{A}} \mathfrak{E}_{h}
$$

Then $\mathfrak{E}$ is an adequate reduction system for $\mathcal{A}$ ([1], Lemma 3.1(1)), and $\mathrm{f}$ reducible implies $\mathfrak{E} \neq \emptyset$, so every element of $\mathcal{A}$ is reducible.

Let $\mathcal{M}_{\mathfrak{E}}(\mathbf{S})$ denote the stabilizer of $\mathfrak{E}$ in $\mathcal{M}(\mathbf{S})$, and let $\Lambda: \mathcal{M}_{\mathfrak{E}}(\mathbf{S}) \rightarrow \mathcal{M}\left(\mathbf{S}_{\mathfrak{E}}\right)$ be the reduction homomorphism. Then $\operatorname{ker}(\Lambda)=\mathcal{D}_{\mathfrak{E}}$, the multitwist group on $\mathfrak{E}$, and thus

$$
\operatorname{ker}\left(\left.\Lambda\right|_{\mathcal{A}}\right)=\operatorname{ker}(\Lambda) \cap \mathcal{A}=\mathcal{D}_{\mathfrak{E}} \cap \mathcal{A}=\mathcal{D}_{\mathfrak{E}} \cap \mathcal{T} \cap \mathcal{A}=\mathcal{T}_{\mathfrak{E}} \cap \mathcal{A} .
$$

We now have a short exact sequence

$$
0 \longrightarrow \mathcal{T}_{\mathfrak{E}} \cap \mathcal{A} \longrightarrow \mathcal{A} \stackrel{\left.\Lambda\right|_{\mathcal{A}}}{\longrightarrow} \Lambda(\mathcal{A}) \longrightarrow 0
$$

of free Abelian groups, which shows that

$$
\operatorname{rank}(\mathcal{A})=\operatorname{rank}\left(\mathcal{T}_{\mathfrak{E}} \cap \mathcal{A}\right)+\operatorname{rank}(\Lambda(\mathcal{A})) \leq \operatorname{rank}\left(\mathcal{T}_{\mathfrak{E}}\right)+\operatorname{rank}(\Lambda(\mathcal{A})) .
$$

We will be done, by applying Lemma 3.2 , once we show that $\operatorname{rank}(\Lambda(\mathcal{A})) \leq \Omega$, the number of components of $\mathbf{S}_{\mathfrak{E}}$ not homeomorphic to a pair of pants or a one-holed torus. 
A theorem of Ivanov ([3], Theorem 1.2) implies that $\Lambda(f)$ restricts to each component $\mathbf{S}_{1}, \mathbf{S}_{2}, \ldots, \mathbf{S}_{\nu}$ of $\mathbf{S}_{\mathfrak{E}}$, giving "projections" $p_{i}: \Lambda(\mathcal{A}) \longrightarrow \mathcal{M}\left(\mathbf{S}_{i}\right)$ induced by restricting representatives. Set $\mathcal{A}_{i}=p_{i}(\Lambda(\mathcal{A})) \subseteq \mathcal{M}\left(\mathbf{S}_{i}\right)$. Then $\Lambda(\mathcal{A}) \subseteq \bigoplus \mathcal{A}_{i}$, so $\operatorname{rank}(\Lambda(\mathcal{A})) \leq \sum \operatorname{rank}\left(\mathcal{A}_{i}\right)$. We make the following observations:

(i) If $\mathbf{S}_{i}$ is a pair of pants, then $\mathcal{M}\left(\mathbf{S}_{i}\right)$ is finite, so $\operatorname{rank}\left(\mathcal{A}_{i}\right)=0$.

(ii) If $\mathbf{S}_{i}$ is a one-holed torus, then the homomorphism $H_{1}\left(\mathbf{S}_{i}\right) \rightarrow H_{1}(\mathbf{S})$ induced by inclusion is injective. Any homeomorphism $f$ representing an element of $\mathcal{A}$ maps a circle $\mathfrak{c}$ in $\mathbf{S}_{i}$ to a circle $\mathfrak{c}^{\prime}$ in $\mathbf{S}_{i}$, so $\mathcal{A}_{i}$ lies within the Torelli group of $\mathbf{S}_{i}$, which is trivial in this case.

(iii) If $\mathbf{S}_{i}$ is neither a pair of pants nor a one-holed torus, then $\mathcal{A}_{i}$ is either trivial or is an adequately reduced torsion-free Abelian subgroup of $\mathcal{M}\left(\mathbf{S}_{i}\right)$. So again by McCarthy's theorem, $\operatorname{rank}\left(\mathcal{A}_{i}\right) \leq 1$.

These observations tell us that

$$
\operatorname{rank}(\Lambda(\mathcal{A})) \leq \sum_{i=1}^{\nu} \operatorname{rank}\left(\mathcal{A}_{i}\right) \leq \Omega .
$$

\section{References}

[1] Joan S. Birman, Alex Lubotzky, and John McCarthy: Abelian and Solvable Subgroups of the Mapping Class Group, Duke Mathematical Journal, Volume 50, Number 4, pp. 1107 - 1120; December 1983.

[2] J. A. Bondy and U. S. R. Murty: Graph Theory with Applications, NorthHolland, New York; 1976.

[3] Nikolai V. Ivanov: Subgroups of Teichmüller Modular Groups, Translations of Mathematical Monographs, Volume 115, American Mathematical Society, 1992.

[4] John D. McCarthy: Normalizers and Centralizers of Pseudo-Anosov Mapping Classes, (preprint), June 8, 1994.

[5] Jerome Powell: Two Theorems on the Mapping Class Group of a Surface, Proceedings of the American Mathematical Society, Volume 68, Number 3, pp. 347 - 350; March 1978.

Department of Mathematics, Michigan State University

East Lansing, MI 48824, USA

Email: vautawwi@pilot.msu.edu

Received: 12 December $2001 \quad$ Revised: 24 February 2002 